\title{
Application of Superconducting Fault Current Limiter in Power Grid - Survey
}

\author{
Vipul Sharma \\ M. Tech. Scholar \\ NRI Institute of Research \& Technology \\ Bhopal, India \\ vipul10511@gmail.com
}

\author{
Mrs. Madhu Upadhyay \\ Head of Department \\ NRI Institute of Research \& Technology \\ Bhopal, India \\ madyant44@gmail.com
}

\begin{abstract}
Introduction of Distributed Energy Sources (DES) is the highest change happening to the distribution network. This paper describes different types of current limiting methods which reduce the magnitude of the fault current. The interconnected distributed energy sources to the conventional grid improves the power generation capacity of the power system but also increases the magnitude of fault current which cannot tolerate by the short-circuit ratings of the circuit breaker, relays, isolator etc. This paper reviews on the innovative electric equipment i.e. Superconducting Fault Current Limiter (SFCL), which reduces fault current magnitude in first cycle of fault current. Keywords: Distributed Energy Sources, Fault current, Superconducting Fault Current Limiter (SFCL), Protection Equipment.
\end{abstract}

Keywords: SFCL, AI techniques, FACTS, Circuit breaker

\section{INTRODUCTION}

The requirement for electricity worldwide, including India, is growing rapidly and the demand for electricity is higher than electricity due to larger homes, population growth, air conditioning, larger televisions, and more computers. To date, many techniques such as higher impedance transformers, bus bars, and fuses have been used in many industries to suppress the size of fault currents.

However, these devices can affect the reliability of the power system and increase the power loss. SFCL is one of the most recent solutions to the problem of increasing leakage current [1] [2]. The fault current levels of an interconnected electricity network have generally increased due to the increase in energy requirements.
This increase in the fault current, if not correctly reduced, can exceed the maximum rated values of the switchgear [3] [4]. Many conventional protective devices such as series reactors, fuses, high resistance transformers, etc. lead to high costs, greater power loss, and loss of stability of the power supply system, which can lead to a reduction in reliability and reduced operational flexibility. The SFCL is a flexible alternative to the use of conventional protective devices because it effectively reduces the fault current during the first fault current cycle, low weight, and zero impedance during normal operation [5].

SFCL research and development is conducted by numerous electrical manufacturers and public utilities for electrical transmission lines. The future installation of SFCL in the transport network requires an assessment of its impact on the coordination between the generator capacity curves and the backup protection of the generator withdrawal phase [6]. In a power system, an SFCL can be placed in different places, e.g. for example (I) entry point (ii) bus connection point (iii) bus coupling.

\section{LITERATURE REVIEW}

Y. Zhang et al. [1] This article presented the art of residual current limiters (FCL), which specialize in devices within the near-field test state or, then assign the various types to the foremost appropriate nodes during a Smart consistent with FCL and Smart capabilities and functionality Grid: 1) FCL semiconductors are often installed on the inputs of micro-grids and renewable 
energy sources to exchange circuit breakers and maintain coordination of the protection of the transport network.

2) FCL resistive superconductor, FCL superconductor with saturated iron core and dynamic FCL are often installed in distribution stations to take care of protection from downstream overcurrent's without disturbing the harmonics; and 3) FCLs with a resistive and saturated superconducting iron core are often installed in optimal locations within the transmission network to scale back fault currents to a tolerable range when a replacement power station is installed.

S. Yadav et al. [2] Electricity demand is increasing rapidly and electricity demand precedes supply. The introduction of distributed energy resources (DES) is that the biggest change within the distribution network. There's a greater integration of DES into the distribution network by means of power electronics converters to satisfy the growing supply needs. The penetration of distributed energy resources should further increase within the future. The connection of the distributed energy resources to the distribution network increases the residual current value, which may cause anomalous conditions throughout the electricity network. The equipment installed within the power station and substation is extremely expensive. It's therefore necessary to guard these devices from fault currents.

H. Kraemer et al. [3] The active a part of the limiter consists of 63 two-wire coils in $12 \mathrm{~mm}$ stabilized YBCO steel conductor and is housed during a cryostat that operates at 5 bar and $74 \mathrm{~K}$. the primary phase has been completely assembled and successfully tested for switching power and high voltage test. Basic system design and test results are provided. The work was partially financed by the US-DOE with the contract number DE-FC26-07NT43243.

J. Kozak et al. [4] a study on superconductive residual current limiters (SFCL) is meant to guard the electricity grid from errors. The rapid increase in SFCL impedance reduces the short current. During this article, we present the planning and development of coreless inductive SFCLs for MV distribution systems. It's a really attractive design that reduces the load of the device because of the coreless construction and therefore the dimensions of the first winding, which is reduced because of the cryogenic cooling. The first $2 \mathrm{G}$ HTS and secondary 2 G HTS windings are magnetically coupled.

\section{APPlications OF SFCL IN POWER SYSTEM}

\section{A. Limit the fault current}

The residual current limiter (FCL) is used to limit very high current at high speed when errors occur. Unlike a normal reactor, the normal impedance is very low and has designed an impedance in a fault situation. The fault limit speed is high enough to limit the fault current in $1 / 4$ cycle. This function must also be restored quickly and automatically. Various FCLs are under development, some of which are used in the fuel system. The most typical FCL is to switch from a low impedance circuit to a high impedance circuit. Circuit breakers and power electronic devices are used to control FCL circuits [5] [7].

A superconducting deficiency flow limiter (SFCL) sequential with a downstream electrical switch may be a practical answer for controlling issue flow levels in electrical circulation systems. The framework contemplates show that SFCL can't just restrain the issue current to a reasonable worth, yet in addition decrease the drop. The transient recuperation voltage (TRV) may be extensively weakened and improved by the nearness of the SFCL in the wake of opening the breaker to address the error. As promising use of superconductors, SFCL is one among the creative gadgets of FACTS inside the power grid.

\section{B. Secure interconnector to the network}

Using SFCL would not only reduce the load on the device, but also provide a connection to protect the network. It is possible to improve the reliability and stability of power systems by reducing the fault current. If the bus bars are coupled via an SFCL, the short circuit power can be doubled. Another improvement can be achieved if low impedance transformers are used in series with SFCL.

\section{Reduces the voltage sag at distribution system}

When the SFCL is installed in the circuit's electrical distribution system, the fault current decreases based on the position and resistance of the SFCL and voltage drops are improved [8]-[12].

\section{Control Technologies}

Non-Superconducting: Fault Current Limiters that don't depend upon Superconducting materials to perform the 
present limiting action. It contains current limiting fuses, solid-state devices \& many other [13].

Superconducting: Fault current Limiters that depend upon Superconducting material to perform.

\section{TYPES OF SFCL}

SFCL is an innovative electrical device that can reduce the size of the fault current during the first half cycle of the fault current. Depending on the application, SFCLs are classified as:-

\section{A. Resistive Superconducting Fault Current Limiter (RSFCL)}

It is simple because the superconductors are series connected with the phase conductors electrically. Resistive SFCL works on the concept that a current is passing in the conductor, when this passing current is more than the rated critical current, IC of superconductor, quenching initiates and this results in a switching to a resistive state.

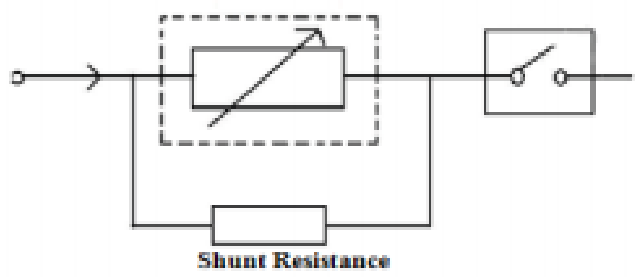

Fig. 1 RSFCL Circuit Diagram

\section{B. Inductive Superconducting Fault Current Limiter (ISFCL)}

It comprise of two iron cores, which are operated by a DC bias supply. Two iron cores are used that can limit the fault current in both directions. The inductive type SFCL has some unique merits like, large design flexibility because of the turn ratio, insulation between a power transmission line, current-limiting devices and low heat losses.

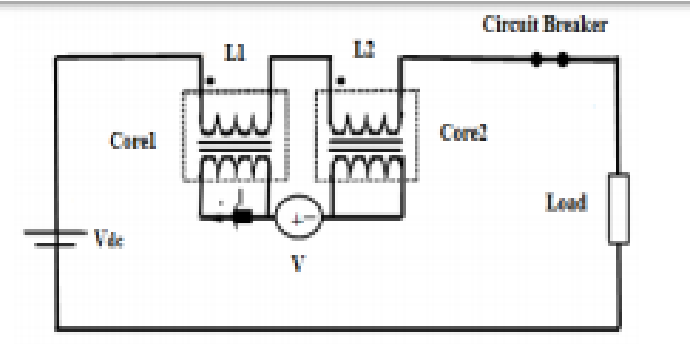

Fig. 2 Inductive SFCL Circuit Diagram
C. Hybrid Superconducting Fault Current Limiter (HSFCL)

In the hybrid type SFCL the fault current is distinguished by a superconductor and bypassed to a reactor in a parallel circuit by a high speed switch for current limitation. HSFCL employs superconductor as a current commutation media and fault current sensor not for current limiting purpose

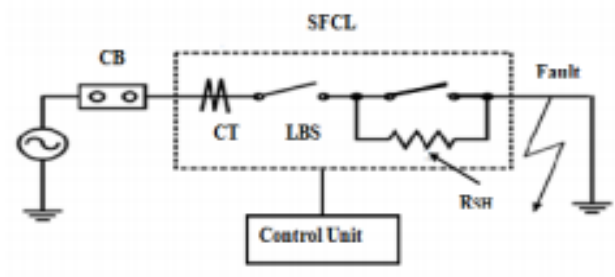

Fig. 3 Hybrid SFCL Circuit Diagram

\section{Problem Formulation}

SFCL is a flexible alternative to the use of conventional protective devices, due to its effective ways of reducing fault current within the first cycle of fault current, reduced weight and zero impedance during normal operation.

The concurrence of fault cases an intensive rise in the voltage at the bus. This can lead to complete cut off of the generation system and also affect its rotor angle steeply as it will cause it to shift to a sudden no load condition.

The SFCL in line with the bus can reduce the fault current to maintain the voltage at the bus and keep the generating system stable and protect it from no load condition.

\section{PROPOSED WORK}

- Need to design a SFCL with having AI based control for its better performance than any other conventional SFCL. This will enhanced system safety, stability, and efficiency of the power delivery systems.

- The device is to be primarily designed for renewable energy systems like solar wind etc.

- The SFCL will be used to design the system for fault analysis and protect the voltage drop at the terminal of generation.

\section{CONCLUSION}

Now a days, a SFCL are very attracted solution to limit the fault current. And in this paper the various 
application of SFCL in the power system are briefly discussed. Superconducting fault current limiters are anticipated as a solution for existing electric networks.

The emerging solutions for fault current limiters are SFCL which has several merits such as low cost, high performance, coordination with conventional systems. Finally, our newly developed SFCL would be promised solutions in order to solve the practical problems of conventional SFCL.

\section{REFERENCES}

[1] Y. Zhang, and R. A. Dougal, "State of the Art of Fault Current Limiters and Their Applications in Smart Grid," IEEE Conf in Power and Energy Society General Meeting, year 2012, pp.1-6.

[2] S. Yadav, G. K. Choudhary, and R. K. Mandal, "Review on Fault Current Limiters," International Journal of Engineering Research \& Technology, Vol. 3, No. 4, pp. 1595-1603, 2014.

[3] H. Kraemer, W. Schmidt, H. Cai, B. Gamble, T. Macdonald, J. Mcnamara, W. Romanosky, N. Lallouet, F. Schmidt, and S. Ahmed, "Superconducting Fault Current Limiter for Transmission Voltage," Supercond. Sci. Technol, Vol. 36, pp. 921-926, 2012.

[4] J. Kozak, M. Majka, T. Janowski, and S. Kozak, "Design and Development of the First Polish Superconducting Fault Current Limiter for MV Distribution Systems," Supercond. Sci. Technol, Vol. 36, pp. 845-848, 2012.

[5] S. Shrivastava, S. Jain, and R. K. Nema, "Distributed generation : Technical Aspects of Interconnection," International Journal on Emerging Technologies, Vol. 1, No. 1, pp. 37-40, 2010.

[6] "Superconducting Fault Current Limiters" Electric Power Research Institute, 2009, Available:http://www.suptech.com/pdf products/f aultcurrentlimiters.pdf

[7] D. Schwanz, M. Bollen, A. Larsson, and H. Kocewiak, "Harmonic mitigation in wind power plants: Active filter solutions," in 2016 17th International Conference on Harmonics and Quality of Power (ICHQP), pp. 220-225, Oct 2016.

[8] N. Dhlamini and S. P. Chowdhury, "The impact of wind farm aggregation techniques for analyzing power system dynamics," in 2015 50th International Universities Power Engineering Conference (UPEC), pp. 1-6, Sept 2015.

[9] J. Y. Ruan, Z. X. Lu, Y. Qiao, and Y. Min, "Analysis on applicability problems of the aggregation-based representation of wind farms considering dfigs x2019; lvrt behaviors," IEEE Transactions on Power Systems, vol. 31, pp. 4953-4965, Nov 2016.

[10] M. Kayikci and J. V. Milanovic, "Assessing transient response of dfigbased wind plants x2014; the influence of model simplifications and parameters," IEEE Transactions on Power Systems, vol. 23, pp. 545- 554, May 2008.

[11] D. Ochoa and S. Martinez, "A simplified electro-mechanical model of a dfig-based wind turbine for primary frequency control studies," IEEE Latin America Transactions, vol. 14, pp. 3614 3620, Aug 2016.

[12] L. P. Kunjumuhammed, B. C. Pal, C. Oates, and K. J. Dyke, “The adequacy of the present practice in dynamic aggregated modeling of wind farm systems," IEEE Transactions on Sustainable Energy, vol. 8, pp. 23-32, Jan 2017.

[13] U. Vargas and A. Ramirez, "Extended harmonic domain model of a wind turbine generator for harmonic transient analysis," IEEE Transactions on Power Delivery, vol. 31, pp. 1360-1368, June 2016. 